\title{
Salvamento de la extremidad pélvica en México: una opción viable para evitar amputaciones
}

\author{
Lower extremity salvage and reconstruction surgery in \\ Mexico, a viable option to avoid amputation
}

\begin{abstract}
Atristain-Carrión Ramiro J, ${ }^{1}$ Rodríguez-Collazo Edgardo R, ${ }^{2}$ Delgado-Pérez José Antonio, ${ }^{3}$ Isunza-Alonso Oscar $D,{ }^{4}$ Migoya-Nuño Alfonso ${ }^{5}$
\end{abstract}

En México, las deformidades congénitas de las extremidades pélvicas representan un reto terapéutico asociado con pronóstico sombrío. ${ }^{1}$ En concreto, las hemimelias tibial y peronea, y las hipoplasias femorales proximales, por su alto costo y complejidad técnica, suelen terminar en amputación o persistencia de la deformidad en la vida adulta. ${ }^{2}$ Ambas situaciones repercuten en la calidad de vida de los pacientes, que se refleja en la falta de integración a la sociedad e incapacidad para tener una vida autosustentable e independiente. ${ }^{3,4}$

De manera global, se estima que la incidencia de deficiencias congénitas de las extremidades pélvicas es de 1-20,0005 (1-50,000 para hemimelia peronea, $1-50,000$ deficiencia femoral proximal y 1-1,000,000 hemimelia tibial). ${ }^{6,7,8}$ En los últimos cinco años, al Instituto Nacional de Pediatría acudieron 30 pacientes a valoración de segunda opinión, porque se les había propuesto la amputación de la extremidad.

Desde el punto de vista histórico, se ha recurrido a las amputaciones ${ }^{9,10}$ porque la reconstrucción requiere múltiples cirugías a largo plazo (incluso 15 años), insumos ortopédicos de alto costo y la participación de un equipo multidisciplinario integrado, al menos, por un ortopedista adiestrado en salvamento de la extremidad pélvica. Tal situación está cambiando por el apoyo de fundaciones: CLESF (Chicago Lower Extremity Surgical Foundation, con sede Chicago, Estados Unidos), cuyo principal objetivo es el salvamento o reconstrucción de las extremidades pélvicas, y que a partir de 2006 se ha encargado de cubrir los costos de tratamiento en México, incluido el material y los recursos necesarios para facilitar la participación de cirujanos con experiencia y la capacitación del personal local, lo que ha permitido que se lleven a cabo diversos procedimientos exitosos. El trabajo en equipo realizado entre el INP y CLESF ha permitido intervenir 24 casos para salvamento de la extremidad pélvica desde el año 2015 a la fecha.
${ }^{1}$ Presidente y cofundador de la Chicago Lower Extremity Surgical Foundation (CLESF), Chicago, EUA.

2 Director del Chicago Foot \& Ankle Deformity Correction Center at Presence Saint Joseph Hospital, Chicago, EUA.

${ }^{3}$ Adscrito al servicio de Ortopedia pediátrica, Instituto Nacional de Pediatría, Ciudad de México.

${ }^{4}$ Ortopedista pediatra, Hospital Ángeles Pedregal, Ciudad de México, Hospital Ángeles Puebla, Puebla.

Este artículo debe citarse como Atristain-Carrión RA, Rodríguez-Collazo $E R$, Delgado-Pérez JA, Isunza-Alonso OD, Migoya-Nuño A. Salvamento de la extremidad pélvica en México: una opción viable para evitar amputaciones. Acta Pediatr Mex. 2109;40(5):253-55. 
En México no existe información relacionada con este problema desde un punto de vista holístico, en el que se tomen en cuenta los aspectos sociales, psicológicos y funcionales de los pacientes, por lo que a continuación reportamos nuestra experiencia, que si bien es heterogénea y la serie no es muy grande, permite en todos los casos evaluar de manera individual la repercusión de la cirugía de salvamento de la extremidad pélvica en estos niños de una manera global; hacemos hincapié en la importancia del efecto psicológico en este grupo de pacientes, para su posterior independencia e integración a la sociedad como adultos productivos.

En el Instituto Nacional de Pediatría se han atendido 24 pacientes, con media de edad de 10 años, 15 de 24 del sexo masculino y 9 de 24 del femenino; 6 de 24 con afectación bilateral, 10 de 24 del lado izquierdo y 8 de 24 del lado derecho, y se requirió una media de 2.5 cirugías para alcanzar el objetivo individual de cada caso.

En la evaluación preoperatoria se interrogó de manera específica a los pacientes o sus padres acerca de las expectativas relacionadas con el tratamiento. Todos respondieron que entre sus expectativas se encontraba la corrección funcional para mejorar el patrón de marcha e independencia para las actividades de la vida diaria y cuidado personal, 17 de 24 incluyeron la mejoría estética, 8 de 24 el control del dolor y 9 de 24 poder usar zapatos.

Al finalizar el tratamiento, 23 de los 24 pacientes se mostraron satisfechos y con los objetivos logrados según sus expectativas planteadas. Hoy día los 24 pacientes continúan en seguimiento médico y rehabilitación, para maximizar su potencial de marcha e independencia. Solo 1 paciente decidió abandonar el tratamiento posterior a la práctica de dos cirugías, al mencionar que no deseaba más intervenciones quirúrgicas ni continuar con el uso de fijación externa.
Los resultados expuestos sugieren llevar a cabo una profunda reflexión de la repercusión de este tipo de lesiones, con la intención de conseguir la independencia y productividad en la vida adulta de los pacientes, por lo que debe realizarse un esfuerzo para el correcto tratamiento de las lesiones porque, si bien es verdad que algunas de estas reciben su máximo beneficio al realizar la amputación y correcta rehabilitación, la mayoría de los pacientes se beneficia con la cirugía de salvamento, como se observó en los 24 pacientes.

Un factor determinante para la toma de decisiones que los padres y pacientes deben considerar al optar por la reconstrucción como salvamento, en vez de la amputación, es el alto costo de las cirugías.

La reconstrucción tiene un costo elevado y casi siempre es inaccesible en nuestro medio, considerando la amputación como única opción, situación que se busca evitar, porque a nuestro parecer (específicamente en nuestro medio, donde culturalmente las amputaciones no son bien aceptadas y la tasa de seguimiento a largo plazo es mala) la reconstrucción representa la mejor opción a largo plazo para la mayoría de los pacientes con hemimelias o deficiencias longitudinales, lo que permite una alta tasa de integración a la sociedad como adultos independientes, económicamente activos. Si bien es cierto que los niños tienen alta capacidad de adaptación a las amputaciones, éstas requieren el uso de prótesis para su funcionamiento correcto, que resultan de igual manera muy costosas y deben reemplazarse periódicamente de acuerdo con el crecimiento y edad del paciente, además de requerir rehabilitación extensa y asociarse con elevada tasa de complicaciones.

En conclusión, estamos convencidos que todo esfuerzo dirigido a lograr la integración de estos niños a la sociedad como adultos autónomos 
debe realizarse, independientemente de si la mejor opción resulta en el salvamento o la amputación y apoyo de prótesis; sin embargo, todos los casos deben evaluarse de manera individual, por un equipo de expertos, que tomen como base para el tratamiento las opciones terapéuticas a las que puede accederse en nuestro medio, las expectativas del paciente y los padres, el medio geográfico en el que ese niño se desenvolverá como adulto, el contexto cultural, el apoyo familiar que tendrá a lo largo del tratamiento y la capacidad física e intelectual del paciente y los familiares para seguir las instrucciones médicas.

\section{REFERENCIAS}

1. Gutiérrez G, et al. Hemimelia peronea. Revisión de la bibliografía a propósito de un caso. Rev Esp Méd Quir 2009;14(3):141-144. http://www.redalyc.org/articulo. oa?id=47312183007

2. Antonio J, et al. Inestabilidad del tobillo secundaria a hemimelia peronea en adulto. Presentación de un caso. Acta Ortop Mex 2006;20(4):172-4. https://www.medigraphic. com/cgi-bin/new/resumen.cgi?IDARTICULO=8324
3. Montpetit K, et al. Measurement of health-related quality of life in children undergoing external fixator treatment for lower limb deformities. J Pediatr Orthop 2009;29(8):920-6. https://doi.org/10.1097/BPO.0b013e3181c1e2e2.

4. Onat SS, et al. The importance of orthoses on activities of daily living in patients with unilateral lower limb amputations. J Back Musculoskelet Rehabil. 2017;30(4):829-833. DOI: 10.3233/BMR-160532.

5. Ghanem I. Epidemiology, etiology and genetic aspects of reduction deficiencies of the lower limb. J Child Orthop 2008;329-32. DOI: 10.1007/s11832-008-0098-9.

6. Navarrete-Hernández E, et al. Malformaciones congénitas al nacimiento. Bol Med Hosp Infant Mex 2017;74(4):301-308. http://dx.doi.org/10.1016/j.bmhimx.2017.02.003

7. Canún $\mathrm{S}$, et al. Prevalencia de malformaciones congénitas de herencia multifactorial de acuerdo con los certificados de nacimiento y muerte fetal. México, 2008-2012. Bol Med Hosp Infant Mex 2015;72(4):290-294. http://dx.doi. org/10.1016/j.bmhimx.2015.06.008

8. Hootnick DR, et al. The syndrome of proximal femur, fibula, and midline metatarsal long bone deficiencies. Birth Defects Res 2018;110(15):1188-1193. DOI: 10.1002/bdr2.1349

9. Reggie $\mathrm{C}$, et al. Congenital fibular deficiency. J Am Acad Orthop Surg 2014;22:246-255. http://dx.doi.org/10.5435/ JAAOS-22-04-246

10. Westberry $D E$, et al. The boyd amputation in children: indications and outcomes. J Pediatr Orthop 2014;34(1):86-91. DOI: 10.1097/BPO.0b013e3182a007a0 